\title{
Evolution of Secondary Electron Emission Characteristics of Spacecraft Surfaces
}

\author{
R.E. Davies* and J.R. Dennison ${ }^{\dagger}$ \\ Utah State University \\ Logan, Utah, 84322-4415
}

\section{Introduction}

Secondary electron emission (SEE) - i.e., the ejection of low-energy ( $\leq 50 \mathrm{eV}$ ) electrons from surfaces as a result of energetic electron bombardment-is a key process in the electrical charging of spacecraft operating in a wide range of orbital regimes. ${ }^{1}$ While severe charging is most often associated with spacecraft operating at geosychronous altitudes, greater-than-kilovolt events have also been reported aboard polar orbiting spacecraft as low as $800 \mathrm{~km}$, and SEE has been identified as one of the dominant mechanisms underlying these events. ${ }^{2}$ Myriad operational anomalies_ranging in severity from minor component disruptions, to temporary loss of vehicle control, to, in at least two very expensive instances, the complete loss of an entire spacecraft-are well-documented consequences of the differential charging of spacecraft components $^{3}$ and of obvious concern to spacecraft designers and controllers. Spacecraft charging models, such as NASA's NASCAP, NASCAP/LEO, and POLAR codes, have been developed to predict charging levels that a given spacecraft may experience based on its geometry, orbit, and the various materials used in its construction. ${ }^{3}$ Critical to the accuracy of these models are estimations of secondary electron (SE) yields (i.e., the numbers of SE's emitted per incident primary electron) for given surfaces under varying conditions of energetic electron bombardment. ${ }^{1}$ SE yields are functions of both material and incident electron energy, and the charging codes incorporate these dependencies via empirically derived SEE models-models requiring, as inputs, experimentally determined yield-vs.-energy curves for a variety of spacecraft materials. ${ }^{3}$ Not presently incorporated into the charging codes, however, are the constantly evolving surface conditions aboard vehicles operating in the space environment, and the effects of such surface evolution on the production of SE's. (These changes result from the continuous removal and addition of surface contaminants as a result of energetic electron, ion, and photon and atomic oxygen bombardment).

Since SEE is primarily a surface phenomenon, SE yields are extremely sensitive to the presence of surface contaminants such as oxide layers and carbon films. From an SEE standpoint, then, the addition or removal of surface contaminants effectively changes the material, resulting in a (sometimes drastically) changing yield- $v$ s.-energy curve. Therefore, as a spacecraft's surfaces evolve, so too do its SEE characteristics and, consequently, its susceptibility to significant charging in a given environment. While this fact has been appreciated qualitatively for a number of years, ${ }^{3}$ the importance of surface conditions and the dynamic evolution of the surface to charging levels has remained largely uninvestigated, and therefore unavailable for incorporation into the charging codes.

\footnotetext{
1*Graduate Research Assistant, Dep't of Physics, Utah State University, UMC 4415, Logan, UT 84322

†Associate Professor, Dep't of Physics, Utah State University, UMC 4415, Logan, UT 84322

R.E. Davies and J.R. Dennison, "Evolution of Secondary Electron Emission Characteristics of Spacecraft Surfaces, “J. Spacecraft and Rockets, 34, 571-574 (1997). DOI: 10.2514/2.3252
} 
The purpose of our investigation, therefore, is twofold: (i) SEE characterization of (conducting) spacecraft materials, subject to varying degrees and types of surface contamination to which operating spacecraft might realistically be subjected, and (ii) investigation of the dynamic evolution of SE yields resulting from energetic electron and ion bombardment of surfaces within a rarefied atmosphere representative of the microenvironment surrounding space vehicles - a region typically contaminated with the by-products of maneuvering thrusters and by the outgassing of non-vacuum-compatible materials on and within the spacecraft. This Note reports experimental results which indicate that contamination and surface dynamics experienced by operating spacecraft may well lead to significant, even drastic, changes in the SEE characteristics of their surfaces. These changes may, in turn, lead to significant underestimations of the charging levels to which a particular vehicle may be subject under a variety of environmental conditions. ${ }^{1}$

\section{Experimental Methods}

Our apparatus allows for the placement of conducting samples into an ultra-high vacuum (UHV) environment, where they are bombarded by energetic (1-5 keV) electrons. Initial contamination of the sample (surface) can be accomplished by surface treatment prior to chamber insertion. Contamination of the vacuum - eventually leading to further contamination of the sample surface through physical or chemical adsorption-can be achieved either by introducing high vapor-pressure materials into the system which will outgas in a UHV environment, or by means of a gas handling system, capable of introducing controlled amounts of contaminant gases . A thorough description of the apparatus and methods employed is presented in Ref. 4.

For the work reported in this Note, a sample of high-purity (4N) polycrystalline aluminum was mechanically polished with $0.3 \mu \mathrm{m}$ alumina powder and cleaned ultrasonically prior to its insertion into a UHV chamber, creating a thin $(\leq \sim 0.1 \mu \mathrm{m})$, smooth aluminum oxide surface reasonably free of bulk surface contaminants. Once inside the vacuum (base pressure $\leq \sim 10^{-10}$ Torr), the sample and chamber were baked at $100^{\circ} \mathrm{C}$ for $\sim 12$ hours. Operating pressures inside the vacuum remained at $\sim 10^{-9}$ Torr and the sample was at room temperature throughout the course of the measurements. The sample was not ion sputtered. Mu-metal shielding surrounding the sample and detection apparatus reduced the ambient magnetic field strength to $\sim 20 \mathrm{mG}$. Contamination of the vacuum resulted from the outgasing of a small piece of PTFE (Teflon)coated wire, placed inside the chamber near the sample. A 200 amu quadrupole mass spectrometer was used for thermal desorption measurements and to monitor residual gas composition. In this contaminated environment, the $\mathrm{Al}$ sample was bombarded with 1-3 keV electrons (produced by a standard Pierce-type electron gun with a tungsten filament) for extended periods (up to 31 hours) and the resulting SE yields monitored as a function of time. The electron beam current density remained stable at $\sim 10^{-3} \mathrm{~A} \cdot \mathrm{cm}^{-2}$. Determination of the SE yields was accomplished via measurement of the net electric current reaching the sample with the sample biased alternately to $0 \mathrm{~V}$ and $+50 \mathrm{~V}$ (details of our measurement technique are given in Ref. 4). Results at $2 \mathrm{keV}$ incident energy, which are representative of repeated trials over an energy range of $1.0 \mathrm{keV}$ to $3.0 \mathrm{keV}$, are presented in Fig. 1. (The size of the circles in the figure is commensurate with the uncertainty in the measurements. Different trials were accomplished by relocating the beamspot on the same sample; reproduction of this experiment on different $\mathrm{Al}$ samples has not yet been attempted.) 


\section{Preliminary Results}

Depicted in Fig. 1(a) is a 2.3-fold monatonic decrease in the SE yield, $\delta$, over a 31-hour period of continuous ( $2 \mathrm{keV}$ ) electron bombardment. Discernible in the data are two general regions of dynamic behavior-labeled Regions I and II in Fig 1(b) — which exhibit different time scales over which changes in $\delta$ are occurring. These results, combined with ancillary measurements described below, suggest the nature of the physical effects leading to the observed evolution of $\delta$ and provide for some measure of quantification of these effects.

The rapid 30\% decrease in $\delta$ in Region I of Fig. 1(b) is likely the result of electron-beaminduced removal from the surface of (i) weakly-bound adsorbed contaminants [Region I-A in Fig. 1(c), occurring on a time scale of seconds], and (ii) more tightly-bound adsorbed contaminants (Region I-B, occurring on a time scale of minutes). This phenomenon-known as electron stimulated desorption (ESD) - is a well-documented result of the energetic electron bombardment of surfaces, ${ }^{6}$ especially familiar to Auger spectroscopists. Residual gas analysis of the chamber atmosphere during this time interval revealed an increased presence (an approximate doubling) of $\mathrm{O}, \mathrm{O}_{2}, \mathrm{CO}$, and $\mathrm{CO}_{2}$ in the chamber atmosphere, consistent with oxide removal due to ESD. Smith ${ }^{7}$ has studied the specific case of $3 \mathrm{keV}$ electrons incident on oxidized $\mathrm{Al}$, and his results are consistent with ours (i.e., the complete desorption of an oxide layer with a comparable current density and on a similar time scale), corroborating the ESD hypothesis. (Note: There is a possibility that some or all of the drop in $\delta_{\text {tot }}(\mathrm{t})$ in Region I-A in Fig. 1 may be due to charging of the oxide layer rather than the dissociation of physisorbed contaminants. Negative charging of the oxide layer could account for a slight rise in the backscattered yield, $\eta$, coincident with the decrease in $\delta$ in the first twelve minutes of the trial. Close examination of the data, however, shows the specific current measurements at $0 \mathrm{~V}$ and +50 $\mathrm{V}$ to be inconsistent with a charging hypothesis, as noted in Ref. 4.)

The continued drop in $\delta$ in Region II following the (presumably) near-complete desorption of the surface contaminants is the result of another well-known electron beam effect-the deposition of disordered carbon, familiar in particular to electron microscopists. ${ }^{8}$ An example of a process known as electron stimulated adsorption (ESA), the build-up of carbon contamination results from the cracking of hydrocarbons (deposited on the surface by the residual gas) by the incident energetic electrons. ${ }^{9}$ Visual inspection, energy dispersive $\mathrm{x}$-ray (EDX) analysis, and $\mathrm{x}$ ray photoemission spectroscopy (XPS) analysis of the sample following its prolonged exposure to the electron beam (and subsequent removal from the UHV chamber) confirmed the deposition of a thin $(\sim 1 \mathrm{~nm})$ carbon film at the beamspot location, and a reduction in the amount of oxygen in the film. ${ }^{4}$ For a $1 \mathrm{~nm}$ thick film deposited over 31 hours, the average deposition rate is seen to be $\sim 0.03 \mathrm{~nm} \mathrm{hr}^{-1}$, or $\sim 0.8 \mathrm{~nm}$ per day.

The above physical interpretation is well modeled with a three-exponential fit to the data of the form

$$
\delta_{\text {tot }}(t)=A e^{-\alpha t}+B e^{-\beta t}+\delta_{A l} e^{-\gamma t}+\delta_{C}\left(1-e^{-\gamma t}\right),
$$

shown in Fig. 1. The terms on the right hand side of this relation are interpreted as the contributions to the total (time-dependent) SE yield, $\delta_{\text {tot }}(\mathrm{t})$, from the weakly-bound contaminants $\left[\mathrm{Ae}^{-\alpha \mathrm{t}}\right]$, the strongly-bound contaminants $\left[\mathrm{Be}^{-\beta \mathrm{t}}\right]$, the bulk aluminum $\left[\delta_{\mathrm{Al}} \mathrm{e}^{-\gamma \mathrm{t}}\right]$, and the deposited 
amorphous carbon film $\left[\delta_{\mathrm{C}}\left(1-\mathrm{e}^{-\gamma \mathrm{t}}\right)\right]$. The constants $\delta_{\mathrm{Al}}$ and $\delta_{\mathrm{C}}$ represent the SE yields for clean (i.e., unoxidized) bulk $\mathrm{Al}$ and bulk disordered carbon, respectively, $\mathrm{A}$ and $\mathrm{B}$ model the SE yields and initial $(\mathrm{t}=0)$ fractional coverages of the weakly-bound and strongly-bound adsorbed species, respectively, and $\alpha, \beta$, and $\gamma$ are time constants describing the periods required for desorption of the weakly-bound species, desorption of the strongly-bound species, and deposition of the carbon layer, respectively. Values for each of these constants, as determined by the fit, are given in Table I and appear reasonable. Specifically, (i) the value for $\delta_{\mathrm{Al}}$ of 0.37 is in fair agreement with that of 0.43 reported by Thomas and Pattinson ${ }^{11}$ for $2.0 \mathrm{keV}$ electrons incident on clean Al; (ii) the value of 0.25 for $\delta_{C}$ is in excellent agreement with a value of 0.27 for soot; ${ }^{13}$ and (iii) values for the time constants are consistent with results reported in Refs. 8 and 9. [Note: In addition to the three-exponential fit given by Eq. (1), two-exponential and four-exponential fits were also attempted. Reduced $\chi^{2}$ analysis, using a least-squares sum divided by the number of degrees of freedon, was used to evaluate the relative success of each of these model functions. The threeexponential fit (seven parameters) given by Eq. (1) provided the best fit, with a reduced $\chi^{2}$ value of 0.348. A two-exponential fit (five parameters) increased the reduced $\chi^{2}$ by $\sim 250 \%$, giving a much poorer fit to the data in all time regimes. A four-exponential fit (nine parameters) decreased the reduced $\chi^{2}$ by $\sim 30 \%$ compared with Eq, (1); however, overparameterization of the data resulted in unacceptably large dependencies (90\% to 100\%) between the parameters. Fitting was performed with a standard analysis package (Sigmaplot, Jandel Scientific) using a Marquardt-Levenberg algorithm. See, for example, Ref. 10.]

\section{Discussion}

The relevance of our experimental results to spacecraft charging calculations depends on the applicability of the laboratory conditions to those of an operating spacecraft; specifically, conditions of pressure, contamination (both species and rate), incident electron energies, and incident electron current densities are considered. We find that (i) the laboratory vacuums employed in our investigation are representative of the low-Earth orbital environment; (ii) laboratory contamination due to outgasing PTFE insulation is representative of the contaminating species found in an operating spacecraft's microenvironment (i.e., the region of space extending from its surface to several tens of meters beyond the vehicle); (iii) laboratory deposition rates of $\sim 1 \mathrm{~nm}$ per day are comparable to deposition rates which have been observed aboard actual space vehicles (discussed below); and (iv) energies of 1-3 keV are common among precipitating auroral electron populations ${ }^{12}$ (several investigations ${ }^{1,2,14}$ have in fact linked severe spacecraft charging events with precipitating electrons of energies $<\sim 4 \mathrm{keV}$ ). Regarding incident electron current densities, however, we find that the current densities employed in our investigation $\left(\sim 10^{-3} \mathrm{~A} \cdot \mathrm{cm}^{-2}\right)$ are much larger than any known natural source currents in the nearEarth environment [where spectral analyses of precipitating auroral electrons ${ }^{12}$ have thus far revealed a maximum integral number flux of $\sim 10^{8} \mathrm{el} \cdot \mathrm{cm}^{-2} \mathrm{~s}^{-1} \mathrm{sr}^{-1}$, equivalent to a maximum current density of $\sim 10^{-10} \mathrm{~A} \cdot \mathrm{cm}^{-2}$ (for a surface exposure of $2 \pi \mathrm{sr}$ )].

Thus the comparisons between laboratory and space conditions are favorable for all relevant parameters save one - electron current density. As a result, we do not expect the SEE evolution due to ESD and ESA observed at $10^{-3} \mathrm{~A} \cdot \mathrm{cm}^{-2}$ in our laboratory to occur at the substantially reduced current densities encountered aboard orbiting spacecraft. Van Oostrom ${ }^{9}$ has demonstrated that no significant ESD of aluminum oxide will occur for total accumulated charge 
dosages of $<10 \mathrm{C} \cdot \mathrm{cm}^{-2}$, and Hillier ${ }^{8}$ has found the ESA rate of carbon to be roughly proportional to the beam intensity for a given energy. The carbon deposition rate also depends, presumably, upon contaminant levels and species in ways which are not yet well understood. Given these data and assuming comparable contaminant levels and a continuous, maximum current density bombardment of $10^{-10} \mathrm{~A} \cdot \mathrm{cm}^{-2}$ (where actual spacecraft are subject to auroral electron bombardment for only a tiny fraction of each orbit, if at all), we estimated $\geq 10^{3}$ years would be required for precipitating electrons to effect significant SEE evolution of a spacecraft surface through the ESD or ESA mechanisms observed in our experiments.

But while ESD and ESA are not likely to effect significant surface modifications aboard space vehicles, other mechanisms do exist which produce similar surface modifications, and with similar concomitant changes in SE yields. Precisely how the surface is modified is not essential; it is the modification itself which is important in determining the SEE evolution of a surface. Results from the LDEF spacecraft in particular have demonstrated that surface modification in the form of the removal and deposition of contaminants does occur on time scales commensurate with a spacecraft's operational lifetime, ${ }^{15}$ and at rates comparable to (and even much greater than) those observed in our investigation. ${ }^{16}$ Crutcher et.al. ${ }^{16}$ report surfaces aboard LDEF with no detectable contamination after 69 months in space, as well as surfaces with contaminant films hundreds of microns thick, translating to deposition rates of up to hundreds of nanometers per day (as compared to $\sim 1 \mathrm{~nm}$ per day in our investigation). Photodissociation of contaminants under vacuum UV bombardment, ion-induced desorption, and attack by atomic oxygen are all ubiquitous mechanisms which remove material, while deposition may result from preferential adsorption of contaminant gases on cooler surfaces, the collection of ionized contaminants on negatively charged surfaces, or atomic oxygen-induced oxidation.

From a spacecraft charging (and SEE) viewpoint, the result of such surface modifications is the same, regardless of the mechanisms involved in producing the modification. In the case of oxidized aluminum, the overall result, based on our measurements, is a significant reduction of the SE yield over time. Since the most troublesome spacecraft potentials are negative, the reduction of a surface's SE yield translates to increased spacecraft-to-plasma charging levels for a given set of environmental conditions. On the other hand, it is interesting to note that carbon deposition occurring over relatively large portions of a spacecraft's surface, encompassing a checkerboard of different materials, may produce, over time, a vehicle whose surfaces are more or less uniform with respect to their SEE characteristics. Such an occurrence may actually reduce the likelihood of significant differential charging between some components.

\section{Conclusions}

Based on our laboratory measurements and the above analysis, it is reasonable to conclude that interactions between operating space vehicles and the space environment can produce surfaces with significantly and even drastically altered SEE characteristics over timescales much less than spacecraft lifetimes. Further, it is important to emphasize that these changes are occuring as a result of the deposition and removal of surface films on the order of only $1 \mathrm{~nm}-$ i.e., tens of atoms - thick. While the ESD and ESA observed in our laboratory are not likely to be the prime mechanisms for the surface modifications which occur on spacecraft surfaces, other mechanisms produce, at comparable rates, surface modifications similar to those observed in this investigation; it is reasonable to assume that the resulting effects on secondary electron yields will be comparable. Of concern to spacecraft designers and controllers should be the fact that

R.E. Davies and J.R. Dennison, "Evolution of Secondary Electron Emission Characteristics of Spacecraft Surfaces, “J. Spacecraft and Rockets, 34, 571-574 (1997). DOI: 10.2514/2.3252 
these changes in SE yields lead to significant changes in a spacecraft's equilibrium potential in a given charging environment. The results of this investigation have clearly demonstrated the need for a systematic, quantitative study of the dynamic evolution of the SEE characteristics of spacecraft surfaces due to space environment effects. Specifically, such study is necessary if present charging codes are to accurately assess the electric potentials to which spacecraft may be subject over their entire operational lifetimes.

\section{Acknowledgments}

This work was supported by fellowships from NASA's Rocky Mountain Space Grant Consortium and by the NASA Graduate Student Researcher's Program. Additionally, we gratefully acknowledge the contributions of Professors John Raitt and Jan Sojka of the Utah State University Physics Department.

\section{References}

${ }^{1}$ Katz, I., Mandell, M., Jongeward, G., and Gussenhoven, M.S., "The importance of accurate secondary electron yields in modeling spacecraft charging," J. Geophys. Res., Vol. 91, No. A12, 1986, pp. 13,739-13,744.

${ }^{2}$ Frooninckx, T.B., and Sojka, J.J., "Solar cycle dependence of spacecraft charging in low Earth orbit," J. Geophys. Res., Vol. 97, No. A3, 1992, pp. 2985-2996.

${ }^{3}$ Garrett, H.B., "The charging of spacecraft surfaces," Rev. Geophys., Vol. 19, No. 4, 1981, pp. 577-616.

${ }^{4}$ Davies, R.E., "An instrument for experimental secondary electron emission investigations, with application to the spacecraft charging problem," MS Thesis, Utah State University, May, 1996.

5Dionne, G.F., "Effects of secondary electron scattering on secondary emission yield curves," J. Appl. Phys., Vol. 44, No. 12, 1973, p. 5361.

${ }^{6}$ Ramsier, R.D., and Yates, J.T. Jr., "Electron-stimulated desorption: principles and applications," Surf. Sci. Rep., Vol. 12, 1991, pp. 247-378.

${ }^{7}$ Smith, T., "Auger electron spectroscopy and ion sputter profiles of oxides on aluminum,: Surf. Sci., Vol. 55, 1976, pp. 601-624.

${ }^{8}$ Hillier, J. "On the investigation of specimen contamination in the electron microscope," $J$. Appl. Phys., Vol. 18, March, 1948, pp. 226-230.

${ }^{9}$ van Oostrom, A., "Some aspects of auger microanalysis," Surf. Sci., Vol. 89, 1979, pp. 615634.

R.E. Davies and J.R. Dennison, "Evolution of Secondary Electron Emission Characteristics of Spacecraft Surfaces, “J. Spacecraft and Rockets, 34, 571-574 (1997). DOI: 10.2514/2.3252 
${ }^{10}$ Titterington, D.M., Smith, A.F.M., and Makov, U.E., in Statistical Analysis of Finite Mixture Distributions, Wiley, New York, 1985, p. 114.

11Thomas, S., and Pattinson, E.B., "Range of electrons and contribution of backscattered electrons in secondary production in aluminum," J. Phys. D: Appl. Phys., Vol. 3, 1970, p. 349.

${ }^{12}$ Hardy, D.A., Gussehnoven, M.S., and Holeman, E., "A statistical model of auroral electron precipitation," J. Geophys. Res., Vol. 90, 1985, pp. 4229-4248.

${ }^{13}$ CRC Handbook of Chemistry and Physics, 65th Edit., edited by R.C. Weast (CRC Press, Inc., Boca Raton, FL, 1984), p. E-352.

${ }^{14}$ Gussehnoven, M.S., and Mullen, E.G., "Geosynchronous environment for severe spacecraft charging," J. Spacecraft Rockets, Vol. 20, No. 1, 1983, pp. 26-34.

${ }^{15}$ Crutcher, E.R., Nishimura, L.S., Warner, K.J., and Wascher, W.W., "Migration and generation of contaminants from launch through recovery: LDEF case history," in LDEF-69 Months in Space, First Post-Retrieval Symposium, NASA Conf. Pub. 3134, Part 1, 1991, pp. 121-140.

${ }^{16}$ Crutcher, E.R., Nishimura, L.S., Warner, K.J., and Wascher, W.W., "Quantification of contaminants associated with LDEF," in LDEF-69 Months in Space, First Post-Retrieval Symposium, NASA Conf. Pub. 3134, Part 1, 1991, pp. 141-177.

*Graduate Research Assistant, Department of Physics

${ }^{\dagger}$ Associate Professor, Department of Physics

Table I. Values for parameters in Eq. (1) for fit of data in Fig. 1.

\begin{tabular}{ccc}
\hline \hline Parameter & Value & Error \\
\hline$\delta_{\mathrm{Al}}$ & 0.370 & $0.6 \%$ \\
$\delta_{\mathrm{C}}$ & 0.250 & $0.7 \%$ \\
$\mathrm{~A}$ & 0.150 & $3 \%$ \\
$\mathrm{~B}$ & 0.06 & $5 \%$ \\
$1 / \alpha$ & 78 seconds & $6 \%$ \\
$1 / \beta$ & 19.6 minutes & $1 \%$ \\
$1 / \gamma$ & 7.7 hours & $6 \%$ \\
\hline \hline
\end{tabular}

R.E. Davies and J.R. Dennison, “Evolution of Secondary Electron Emission Characteristics of Spacecraft Surfaces, “J. Spacecraft and Rockets, 34, 571-574 (1997). DOI: 10.2514/2.3252 


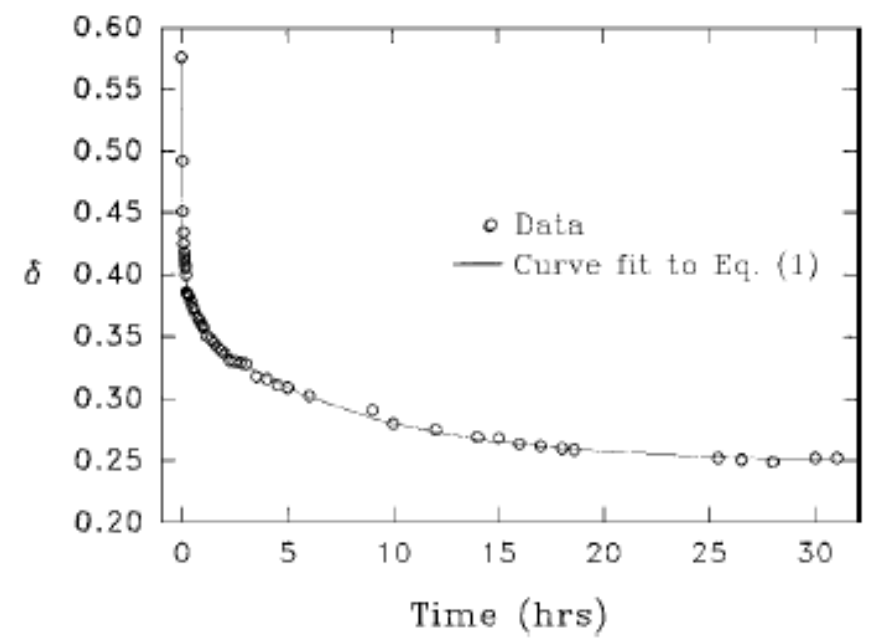

a) Entire 31-h trial

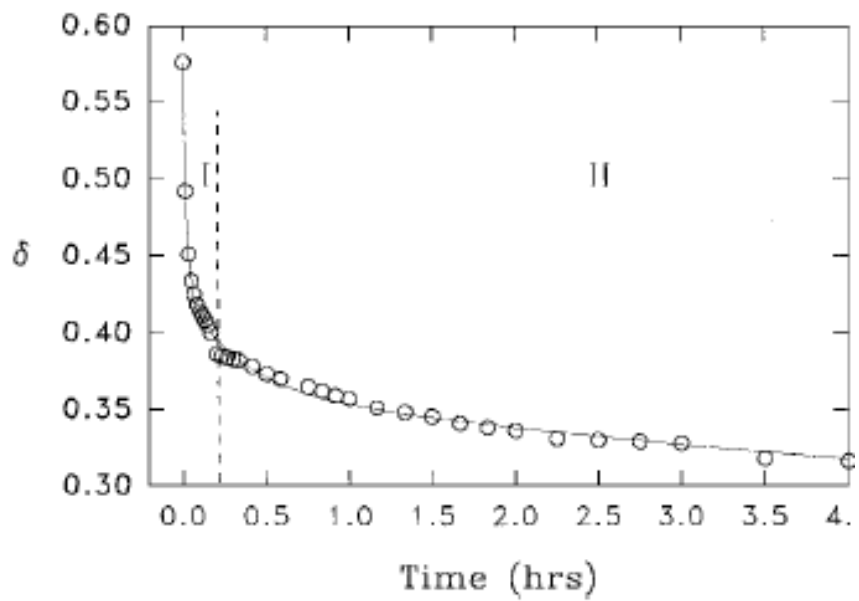

b) First $4 \mathrm{~h}$ of the trial

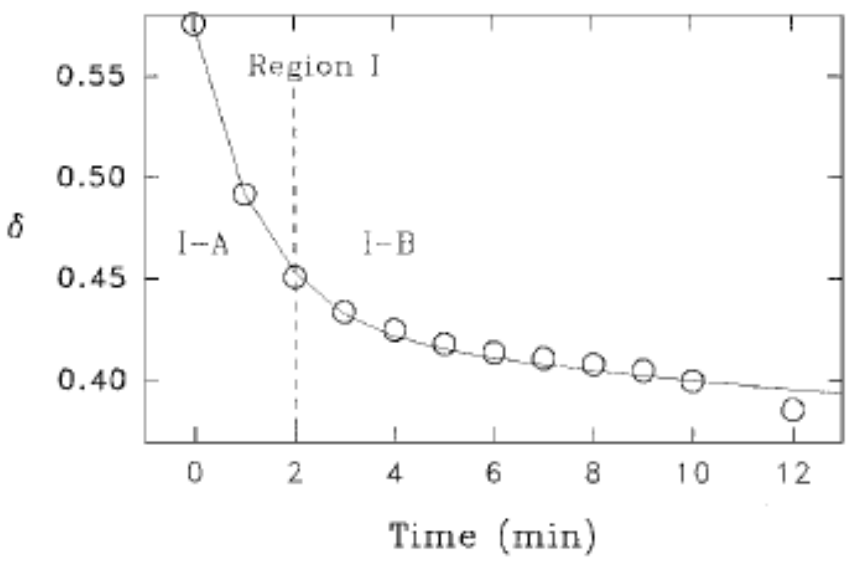

c) First 12 min of the trial

Fig. 1 Secondary yield vs. time for $2.0 \mathrm{keV}$ electrons continuously incident on contaminated aluminum surface: (a) the entire 31-hour trial, (b) the first four hours of the trial, and (c) the first twelve minutes of the trial. 